\title{
Author Correction: Cytotoxic and regulatory roles of mucosal-associated invariant T cells in type 1 diabetes
}

Ophélie Rouxel, Jennifer DaSilva, Lucie Beaudoin, Isabelle Nel, Céline Tard, Lucie Cagninacci, Badr Kiaf, Masaya Oshima, Marc Diedisheim, Marion Salou, Alexandra Corbett, Jamie Rossjohn, James McCluskey, Raphael Scharfmann, Manuela Battaglia, Michel Polak, Olivier Lantz, Jacques Beltrand and Agnès Lehuen

Correction to: Nature Immunology 18, 1321-1331 (2017); published online 9 October 2017.

In the version of this Article originally published, the asterisks indicating statistical significance were missing from Supplementary Figure 6; the file with the correct figure is now available.

\section{Author Correction: Charles D. Surh 1960-2017}

Janko Nikolich-Žugich

Correction to: Nature Immunology 18, 1273 (2017); published online 16 November 2017.

In the version of this article initially published, the year of birth provided (1960) was incorrect. The correct year is 1961 . The error has been corrected in the HTML and PDF versions of the article.

\section{Author Correction: A gammaherpesvirus provides protection against allergic asthma by inducing the replacement of resident alveolar macrophages with regulatory monocytes}

Bénédicte Machiels, Mickael Dourcy, Xue Xiao, Justine Javaux, Claire Mesnil, Catherine Sabatel, Daniel Desmecht, François Lallemand, Philippe Martinive, Hamida Hammad, Martin Guilliams, Benjamin Dewals, Alain Vanderplasschen, Bart N Lambrecht, Fabrice Bureau and Laurent Gillet

Correction to: Nature Immunology 18, 1310-1320 (2017); published online 17 October 2017

In the version of this article initially published, the accession code for the RNA-seq data set deposited in the NCBI public repository Sequence Read Archive was missing from the 'Data availability' subsection of the Methods section. The accession code is SRP125477. 